\title{
Optical Determination of Morphology of Nanoparticles
}

\author{
Bennett B. Goldberg ${ }^{1,2}$, Abdulkadir Yurt ${ }^{3}$, George Daaboul ${ }^{2}$, and M. Selim Ünlü ${ }^{1,2,4}$
}

${ }^{1}$ Department of Physics; ${ }^{2}$ Department of Biomedical Engineering; ${ }^{3}$ Division of Materials Science and Engineering; and ${ }^{4}$ Department of Electrical and Computer Engineering; Boston University, Boston, MA 02215

Using an interferometric imaging technique with a polarization control we demonstrate accurate determination of absolute size and aspect ratio of nanoparticles. The accuracy and highthroughput of the technique is shown through imaging gold nanorods and nanospheres with nonuniform morphology. The method is a powerful tool for high-throughput characterization of anisotropic nanoparticles for biomedical, materials engineering and plasmonics applications.

High-resolution imaging techniques, such as electron microscopy, can accurately determine the shape of nanoparticles but typically require sophisticated equipment and sample preparation and thus have limited throughput. In the optical domain, the spectral signature of the surface plasmon resonance (SPR) response has also shown that aspect ratio of a single gold nanorod can be estimated, but again with limited throughput due to long integration time for detection and SPR is applicable exclusively to plasmonic materials. Here, a widefield, common path interferometer detects single nanoparticles and accurately measures absolute particle dimensions and lateral orientations over a very large sensing area, hence with very high-throughput.

In this talk we discuss and demonstrate a new capability by extending our recent interferometric single-virus detection technique [Daaboul et al., Nano Letters, October 2010] with polarization control as illustrated in Fig.1. The technique utilizes a silicon wafer substrate with a precise layer of oxide that enhances the signal and contrast for a desired range of size and aspect ratio of nanoparticles. We compare this interferometric imaging directly to SEM measurements and verify the accuracy (see Fig. 2). For gold nanoparticles ( $>35 \mathrm{~nm}$ diameter), the accuracy of our measurements is in agreement with SEM measurements within 5nm. The high-throughput capability of our technique is illustrated in Fig. 3 where we display the measured dimensions of the short and long axis of a total of $\sim 230$ nanorods and $~ 130$ nanospheres acquired from single images.

This interferometric imaging technique can be applied to dielectric particles allowing for shape recognition of viral pathogens, providing an orthogonal classification method for specific detection. It is also is easy to implement and readily made portable for point-of-care and resource limited settings. 
(a)

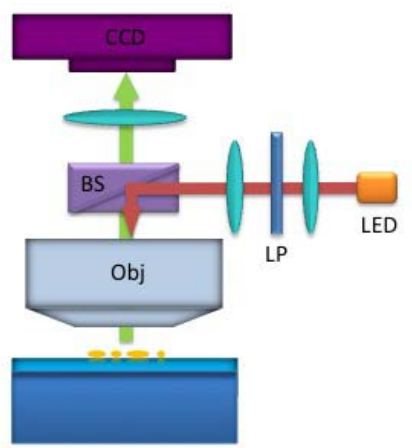

(b)

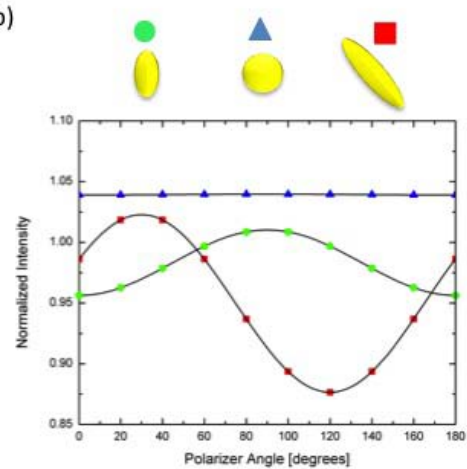

Figure 1: (a) Optical Setup. (b) Simulated peak interferometric response of 3 different nanoparticles (See top view on top panel: circle $\left(d_{1}=25 \mathrm{~nm}\right.$, $\left.d_{2}=50 \mathrm{~nm}\right)$, triangle $\left(d_{1}=45 \mathrm{~nm}, d_{2}=46 \mathrm{~nm}\right)$ and rectangle $\left(d_{1}=30 \mathrm{~nm}, d_{2}=\right.$ $75 \mathrm{~nm})$ ) as a function polarizer angle.

(a)

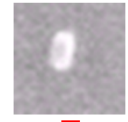

口

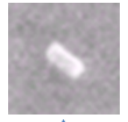

$\Delta$

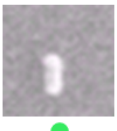

○

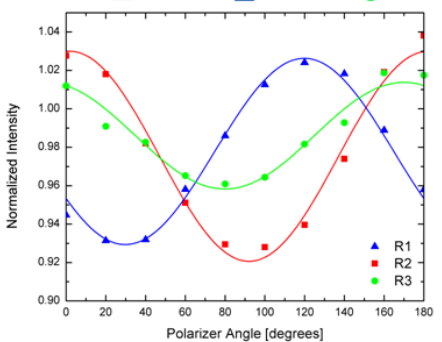

(b)
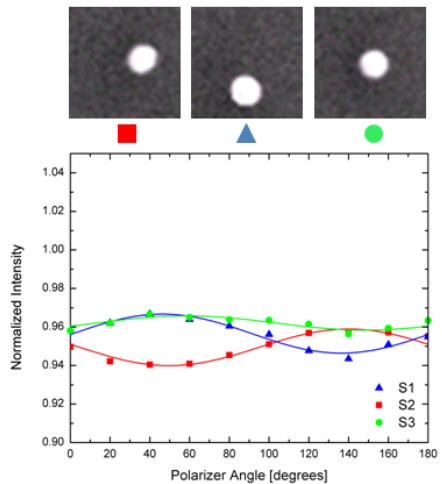

Figure 2: Comparison of SEM and optical measurements of size, aspect ratio and orientation. In (a), SEM micrographs of three nanorods (top panel) and normalized intensity of the same particles is plotted as a function of polarization angle for each particle (bottom panel). (b) the same for three nanospheres.
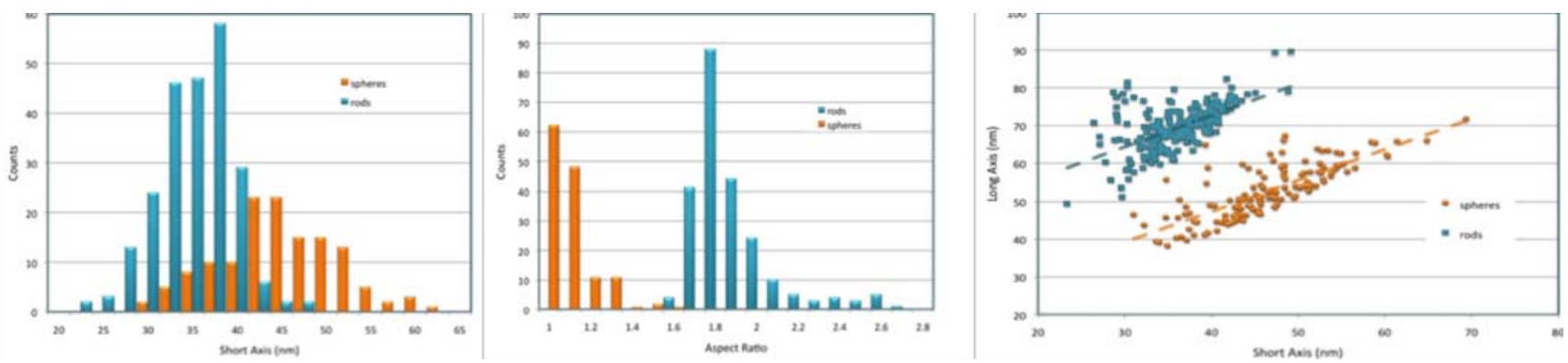

Figure 3: High-throughput measurement results of sample-A (rods) and sample-B (spheres). Histograms for short axis (left) and aspect ratio (middle) are illustrated for sample-A and sample-B. Optical study of individual nanoparticles. (a) Scatter plot showing the short and long axis dimensions of nanoparticles on sample-A (squares) and sample-B (circles). 